www.jmscr.igmpublication.org Impact Factor 5.84

Index Copernicus Value: 83.27

ISSN (e)-2347-176x ISSN (p) 2455-0450 crossref DOI: _https://dx.doi.org/10.18535/jmscr/v5i7.148

\title{
Precancerous Risk Assessment of the Gastric Atrophy by Tri-modal Endoscopy
}

\author{
Authors

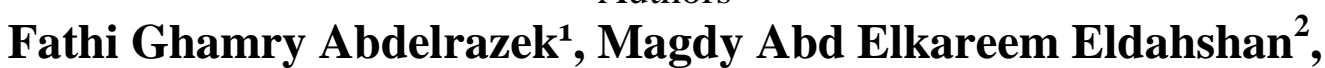 \\ Shadia Hussein Mabrouk ${ }^{3}$, Atef Aboelfotoh Ibrahim ${ }^{4}$, Mohamed Salah Ali Hussein ${ }^{5}$, \\ Mohamed Samy EL Shahawy ${ }^{6}$, Zakaria Mohamed Zakaria ${ }^{7}$, Ahmed Ibrahim Gawish ${ }^{8}$ \\ 1,2,4,5,6,7,8 Gastroenterology Unit, Internal Medicine Dept, Faculty of Medicine, AL-Azhar University, Cairo, \\ Egypt \\ ${ }^{3}$ Pathology Department, Faculty of Medicine, Ain Shams University, Cairo, Egypt \\ ${ }^{1}$ Professor and Head of Gastroenterology Unit, Internal Medicine Dept, Faculty of Medicine, Al Azhar \\ University \\ Email: dr_fathyelghamry@yahoo.com \\ ${ }^{2}$ Professor of Internal Medicine Dept, Faculty of Medicine, Al Azhar University \\ Email: drmagdy1@ hotmail.com \\ ${ }^{3}$ Professor of Pathology Department, Faculty of Medicine, Ain Shams University \\ ${ }^{4}$ Professor of Internal Medicine Department, Faculty of Medicine, Al Azhar University \\ Email: professoratef2012@gmail.com \\ ${ }^{5}$ Assistant Professor of Internal Medicine Department, Faculty of Medicine, Al Azhar University \\ Email: dr_msalahali@yahoo.com \\ ${ }^{6}$ Lecturer of Internal Medicine Department, Faculty of Medicine, Al Azhar University \\ ${ }^{7}$ Lecturer of Internal Medicine Department, Faculty of Medicine, Al Azhar University \\ Email: zakaryashady96@yahoo.com \\ ${ }^{8}$ Resident of Internal Medicine Department, Faculty of Medicine, Al Azhar University \\ Email: dr.ahmedgawish1988@gmail.com \\ Corresponding Author \\ Mohamed Samy EL Shahawy \\ Email: tep_alshahawy@yahoo.com
}

\section{ABSTRACT}

Background / Aims: Gastritis staging and detection of pre-neoplastic gastric mucosal changes based on the histology of biopsy specimen imposes a considerable burden on endoscopist and pathologist. Therefore, we attempted to evaluate whether gastritis staging system based on the using of conventional highresolution white light endoscopy(WLE), Auto fluorescence imaging(AFI), and narrow band imaging(NBI) is equivalent to that determined by histopathology.

Methods: This study included 63 egyptian patients who were diagnosed with chronic gastritis. The lesser curvature of the antrum ( $2 \mathrm{~cm}$ from the pyloric ring) and the lesser curvature of the lower body $(4 \mathrm{~cm}$ from the angle) were examined in consecutive order first by high resolution WLE followed by AFI and finally by NBI. Two endoscopic biopsies were taken from gastric antrum and lower gastric body for histopathological assessment of $H$. Pylori infestation, gastric atrophy and intestinal metaplasia (IM).The lower gastric body 
atrophy was graded according to OLIGA scoring system (Operative Link for Gastritis Assessment) Additionally, the presence or absence of IM was examined in both lower gastric body and antrum.

Results: By means of WLE and AFI, the patients were found to have no, mild, moderate and severe gastric atrophy in 8(16\%), 18(36\%), 8(16\%), and 16(32\%) respectively. Furthermore, Gastric atrophy was detected in all patients by NBI with mild, moderate and severe atrophy in 15(30\%), 24(48\%) and 11(22\%) respectively. as a result of histopathological assessment, 12(24\%), 34(68\%), and 4(8\%) had scores 1, 2 and 3 by OLIGA scoring systems respectively. in view of scoring system of gastric atrophy and intestinal metaplasia, New staging system for gastritis risk assessment was correctly identified Furthermore, Gastric cancer risk stages were recognized and the degree of correspondence on the basis of NBI and combined WLE , AFI on one hand and histopathology on the other hand were $80 \%$ and $68 \%$.

Conclusion: This study demonstrates that staging of premalignant gastric lesions with conventional NBI has better correspondence with histopathology compared with WLE and AFI and can be used for screening early malignancies of the stomach.

Keywords: Narrow-band imaging, Auto-fluorescence imaging, Intestinal metaplasia, white -light endoscopy, Gastric atrophy.

\section{Introduction}

Gastric cancer (GC) is the third most common cause of cancer deaths worldwide ${ }^{[1]}$. Although advanced GC associated with poor prognosis and high mortality rates, early detection and treatment can result in 5-year survival rates as high as $96 \%$ [2]. Secondary prevention through diagnosis of premalignant lesions and early gastric cancer and screening of individuals at high risk are probably the most immediate strategies for improving survival. Patients who have established precursor conditions such as mucosal atrophy or IM are at high risk for developing GC ${ }^{[3]}$. Atrophy of the gastric mucosa is the endpoint of chronic gastritis and represents a precursor condition for gastric cancer. A Dutch nationwide cohort study indicated that the annual incidence of GC was $0.1 \%$ for patients with atrophic gastritis, within 5 years after diagnosis ${ }^{[4]}$. Therefore, identification of patients with gastric atrophy and their followup screening for early GC is fundamental in countries with medium to low incidence of GC, like Egypt ${ }^{[5]}$. In the West, the OLGA (Operative Link for Gastritis Assessment) ${ }^{[6]}$ and OLGIM (Operative Link for Gastric Intestinal Metaplasia assessment) staging systems ${ }^{[7]}$ have become widely used. Both systems assess the risk for gastric cancer on the basis of several biopsy samples taken from the antrum and corpus. The OLGA staging system is based on the severity and topography of atrophy, whereas the OLGIM staging system is based on the severity and topography of intestinal metaplasia ${ }^{[7]}$. In Japan, on the other hand, narrow-band imaging (NBI) endoscopic diagnosis of inflammation, atrophy, and IM of the gastric mucosa has been reported to be fairly accurate ${ }^{[8]}$. If the staging of gastritis based on conventional WLE, AFI and NBI endoscopy approximated to that based on histology of biopsy specimens, it would be as practical as optical biopsy and reduce the work burden of endoscopists, as well as medical costs and procedure time.

\section{Patients and Methods \\ Patients}

This study was conducted at Endoscopy Unit, internal medicine Department of El- Hussein university hospital, AL-Azhar University, cairo, Egypt between December 2015 and November 2016. In this study, Sixty-three (63) Egyptian patients with chronic gastritis were consecutively enrolled. Patients with prior gastric surgery involving the antrum and/or lower gastric body, and patients with portal hypertension were excluded. For each patient, full medical history, along with complete physical examination was conducted. Informed consent was obtained from all subjects.

\section{Endoscopy system}

The instruments used in this study were a videoendoscope and an electronic endoscopic system (Olympus EVIS Lucera Ellit CV-290; Olympus Medical Systems, Tokyo, Japan) and equipped 
with three imaging modes: high-resolution WLE, AFI, and NBI. Mode switch from WLE to NBI or AFI was controlled by buttons on the control head.

\section{Study Design}

In this study, All patients were offered conscious sedation with intravenous Midazolam (2.5-5 mg) and/or Propofol (40-200mg) and then underwent endoscopic assessment for the presence of regular arrangement of collecting venules (RAC), gastric atrophy, and IM in lower gastric body and antrum. To minimize the variability in the endoscopy procedure (macroscopic assessment and biopsy sampling protocol), all of the endoscopic examinations were performed by only one senior endoscopist with more than 10 years experiences in diagnostic and therapeutic endoscopy. The lesser curvature of the antrum $(2 \mathrm{~cm}$ from the pyloric ring) and the lesser curvature of the lower body ( $4 \mathrm{~cm}$ from the angle) were examined in consecutive order first by high resolution WLE, followed by AFI and finally by NBI during single endoscopic examination and scores for lower gastric body atrophy and antral IM was obtained.

The dominant mucosal patterns in each of the two areas were chosen, and photographs were taken after meticulous observation for detection of any lesions. Two biopsy samples were taken from each of these two areas that photographed for histopathological assessment of $\mathrm{H}$. Pylori infestation, gastric atrophy and IM. The entire endoscopic procedure was recorded on a DVD. NBI endoscopic scoring was performed by one endoscopist in two occasions, in the first occasion scoring was done during real time endoscopy, in the second occasion the same endoscopist has performed scoring on the basis of the pictures and videos obtained and the concordance of the scores then was confirmed. The endoscopic scoring system of gastric atrophy and IM was shown in (Table 1). Wight light endoscopy diagnosis and scoring of gastric atrophy was based on Kimura and Takemoto $1969^{[9]}$ classification (Table 1). Moreover, the assessment of gastric atrophy by NBI was based on Alaboudy et al., $2011^{[10]}$ with little modification. In fact Alaboudy et al., originally classified the gastric atrophy and IM into 4 grades following modified Sydney classification. In the current study both advanced and severe grades according to Alaboudy et al., classification of gastric atrophy merged as severe gastric atrophy to meet OLIGA score 3. By means of AFI, the mucosa of the gastric body normally appears purple in the background during examination and if atrophic gastritis is present, the area will appear bright green . As regard to IM , white light endoscopy diagnostic criteria was defined as whitish color change with plaques, patches, or homogeneous discoloration on the gastric mucosa. Additionally, NBI diagnostic criteria of IM defined as a villous or ridge micromucosal pattern with a bright rim (light blue crest) [10] and/or the presence of white opaque substance [11]. Furthermore, AFI diagnostic criteria for IM was defined as any localized patchy lesions that were different in color from the surrounding mucosa ${ }^{[12]}$. OLIGA scoring for gastric atrophy was done for the lower gastric body. As well, the presence or absence of IM was examined in both lower gastric body and antrum. The degree of correspondence between the scores obtained by endoscopy and those obtained from biopsy specimens was determined. By means of the scores obtained by endoscopy and biopsy specimens based on the lower gastric body atrophy OLGA and antral IM, New staging system for gastritis risk assessment was correctly identified (Table 2). The stages were divided into two groups; low risk (stages 0, I, II) and high risk (stages III, IV) and the concordance of gastric cancer risk assessment between histopathology and endoscopy was determined. The diagnosis of H. Pylori and autoimmune gastritis based on the detection of $\mathrm{H}$. Pylori organism in gastric tissue by histopathology, and detection of anti-parietal cell antibodies in serum by Enzyme Linked Immune Sorbent Assay (ELISA), respectively. The endoscopic (WLE and NBI) diagnosis of $\mathrm{H}$. Pylori infection assessed according to presence or absence of regular arrangement of collecting venules (RAC) in gastric body. Patients with 
abscent RAC in upper and/ or lower gastric body were concluded to have $\mathrm{H}$. Pylori infection.

\section{Histology study}

The biopsy samples were fixed in formalin and labeled according to their topographic site (antrum or corpus). Histological sections were obtained from each paraffin block and stained with hematoxylin and eosin. Histological scoring was performed by an expert pathologist blinded to any clinical information. The lower gastric body atrophy was graded according to OLIGA scoring system ${ }^{[7]}$. Furthermore, the presence or absence of IM was depicted in both lower gastric body and antrum.

\section{Statistical Analysis}

Data were analyzed using IBM $\odot$ SPSS $\odot$ Statistics version 23 (IBM@ Corp., Armonk, NY, USA). Normality of numerical data distribution was examined using the D'Agostino - Pearson test. Non-normally distributed numerical variables were presented as mean and standard deviation, Kruskal-Wallis test (for multiple non-ranked group comparisons), or the Jonckheere-Terpstra trend test (for multiple ranked group comparisons). The Conover post hoc test was used for pair wise comparisons if the Kruskal-Wallis or Jonckheere-Terpstra test revealed a statistically significant difference among the groups. Categorical variables were presented as number and percentage and differences were compared using Fisher's exact test.

\section{Results}

Sixty-three patients were recruited. 13 patients were excluded for the following reasons; 3 patients had gastric malignancy, and 10 patients have insufficient biopsy. Therefore, only 50 patients fulfilled the inclusion criteria and included in the current study. Among fifty (50) patients with chronic atrophic gastritis, $29(58 \%)$ were females and 21(42\%) were males; their mean age was $38.7 \pm 15.6$ years (range from 1474 years). 15 patients $(30 \%)$ had history of smoking and 5 patients (10\%) had history of diabetes mellitus. The indication of upper endoscopy was epigastric pain (76\%), heart burn
(10 \%), vomiting (6\%), anemia of unknown etiology (6\%), and dysphagia (2\%), . the etiology of chronic gastritis was related to autoimmune gastritis in 5 patients $(10 \%)$, duodeno-gastric biliary reflux in 8 patients $(16 \%)$ and $\mathrm{H}$. Pylori related gastritis among the remaining 37 patients (74\%). As regard to gastric atrophy and by means of WLE and AFI, patients were found to had no, mild, moderate and severe gastric atrophy in $8(16 \%), 18(36 \%), 8(16 \%)$, and $16(32 \%)$ respectively . Furthermore, Gastric atrophy was detected in all patients by NBI with mild, moderate and severe atrophy in $15(30 \%), 24(48 \%)$ and $11(22 \%)$ respectively (Figure 1). On histopathological assessment of lower gastric body 12 (24\%), 34(68\%), and 4(8\%) had scores 1 , 2 and 3 by OLIGA scoring systems respectively (Figure 2). Pseudopyloric metaplasia was detected in $38(76 \%)$ patients and the frequency increased parallel to the gastric atrophy score detected by NBI . Therefore, $60 \%$ (9/15), $79.16 \%(19 / 24)$ and $90.9 \%(10 / 11)$, of patients with mild, moderate and severe gastric atrophy by NBI had pseudopyloric metaplasia respectively .With histopathological assessment, IM was detected in lower gastric body in 4 patients $(8 \%)$ and antrum in 9 patients (18\%). Additionally, IM was detected by WLE and AFI in lower gastric body in $100 \%$ (4/4), and antrum 33.3\% (3/9) . Resting on NBI, intestinal metaplasia was detected in lower gastric body in $100 \%$ (4/4) and antrum in $44.4 \%$ (4/9). The Overall rate of correspondence between the scores obtained by NBI endoscopy and by OLIGA score in the assessment of lower gastric body atrophy was 58\% (29/50) (Table 3). Moreover, The correspondence between NBI endoscopy and histopathology in diagnosis of antral IM was $86 \%$ (43/50). The histopathological staging of gastric atrophy and Staging by NBI endoscopy was identified and the correspondence between them was $58 \%$ (29/50) (Table 4). The overall rate of correspondence between the scores obtained by combined WLE, AFI and OLIGA score for assessment of lower gastric body atrophy was $28 \% \quad(14 / 50)$ (Table 3). Furthermore, The correspondence between combined WLE, AFI 
and histopathology in diagnosis of antral IM was 84\% (42/50) (Table 3). The histopathological staging of gastric atrophy and Staging by WLE and AFI was identified and The correspondence between them was $30 \%(15 / 50)$ (Table 4). By means of the scores obtained by endoscopy and biopsy specimens based on the lower gastric body atrophy (OLGA) and antral IM , New staging system for gastritis risk assessment was correctly identified (Table 2) and Gastric cancer risk stages were divided into two groups; low risk (stages 0, I, and II) and high risk (stages III and IV) and the degrees of correspondence between the groups determined on the basis of NBI and combined WLE and AFI on one hand and histopathology on the other hand were $80 \%(40 / 50), 68 \%(34 / 50)$ respectively (Table 5).

Table 1 : The endoscopic scoring system of gastric atrophy and intestinal metaplasia

\begin{tabular}{|c|c|c|c|c|}
\hline & $\begin{array}{c}\text { Score } \\
0\end{array}$ & $\begin{array}{c}\text { Score } \\
1\end{array}$ & $\begin{array}{c}\text { Score } \\
2\end{array}$ & $\begin{array}{c}\text { Score } \\
3\end{array}$ \\
\hline & No & Mild & Moderate & Sever \\
\hline $\begin{array}{l}\text { Kimura-Takemoto } \\
\text { classification }\end{array}$ & $\mathrm{CO}$ & $\mathrm{C} 1$ and $\mathrm{C} 2$ & $\mathrm{C} 3$ and $\mathrm{O} 1$ & $\mathrm{O} 2$ and $\mathrm{O} 3$ \\
\hline \multicolumn{5}{|c|}{ Narrow band imaging endoscopy scores of lower gastric body atrophy. } \\
\hline & $\begin{array}{c}\text { Score } \\
0\end{array}$ & $\begin{array}{c}\text { Score } \\
1\end{array}$ & $\begin{array}{c}\text { Score } \\
2\end{array}$ & $\begin{array}{c}\text { Score } \\
3\end{array}$ \\
\hline & No & Mild & Moderate & Sever \\
\hline $\begin{array}{l}\text { RAC (Regular arrangement } \\
\text { of Collecting venules) }\end{array}$ & Present & Absent & Absent & Absent \\
\hline Gastric pits & Not seen & $\begin{array}{l}\text { Rounded or } \\
\text { oval }\end{array}$ & Elongated & $\begin{array}{c}\text { Ground glass } \\
\text { appearance }\end{array}$ \\
\hline Intestinal metaplasia & No & No & No & Yes or No \\
\hline \multicolumn{5}{|c|}{ Narrow band imaging endoscopy scores of antral intestinal metaplasia } \\
\hline \multicolumn{2}{|l|}{ Score 0} & \multicolumn{3}{|l|}{ Score 1} \\
\hline \multicolumn{2}{|c|}{ No light blue crest or white opaque } & \multicolumn{3}{|c|}{ Light blue crest or white opaque substance. } \\
\hline
\end{tabular}

Table 2 : New staging system for gastritis risk assessment

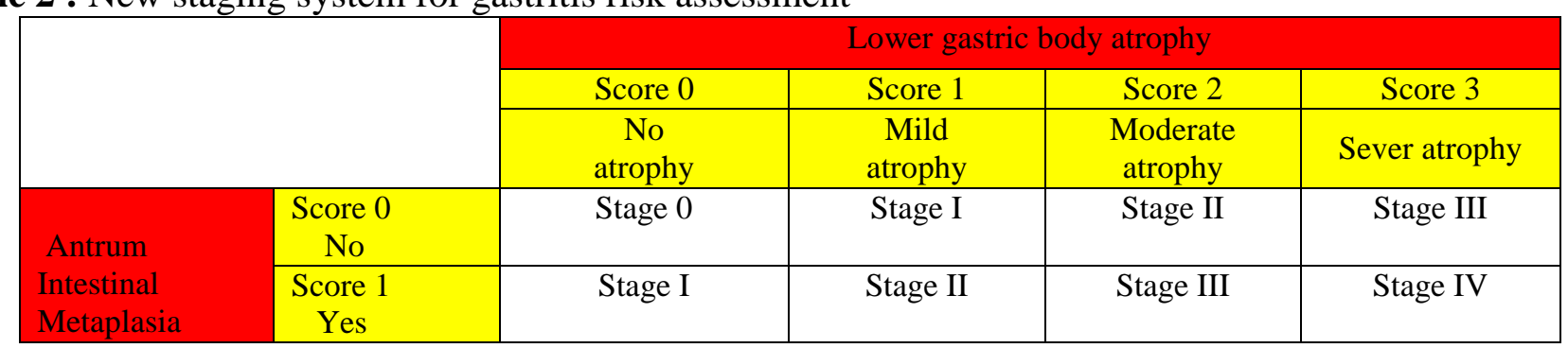

Low risk: stages 0 , I and II

High risk: stages III and IV

Table 3 : The correspondence between endoscopy and histopathology in diagnosis of lower body atrophy and antral intestinal metaphase

\begin{tabular}{|l|l|c|c|c|c|}
\hline \multicolumn{1}{|l|}{ The correspondence between endoscopy and OLIGA score in diagnosis of lower body atrophy } \\
\hline & & \multicolumn{4}{|c|}{ Lower body OLGA score } \\
\cline { 3 - 6 } & & Score 0 & Score 1 & Score 2 & Score 3 \\
\cline { 3 - 6 } & & $\begin{array}{c}\text { Number } \\
(\%)\end{array}$ & Number (\%) & $\begin{array}{c}\text { Number } \\
(\%)\end{array}$ & $\begin{array}{c}\text { Number } \\
(\%)\end{array}$ \\
\hline \multirow{2}{*}{$\begin{array}{l}\text { Lower body } \\
\text { atrophy } \\
\text { WLE,AFI }\end{array}$} & by & 0 & $4(50 \%)$ & $4(50 \%)$ & 0 \\
\cline { 2 - 6 } & mild & 0 & $4(22 \%)$ & $13(72.2 \%)$ & $1(5.6 \%)$ \\
\cline { 2 - 6 } & Moderate & 0 & $1(12.5 \%)$ & $7(87.5 \%)$ & 0 \\
\hline
\end{tabular}




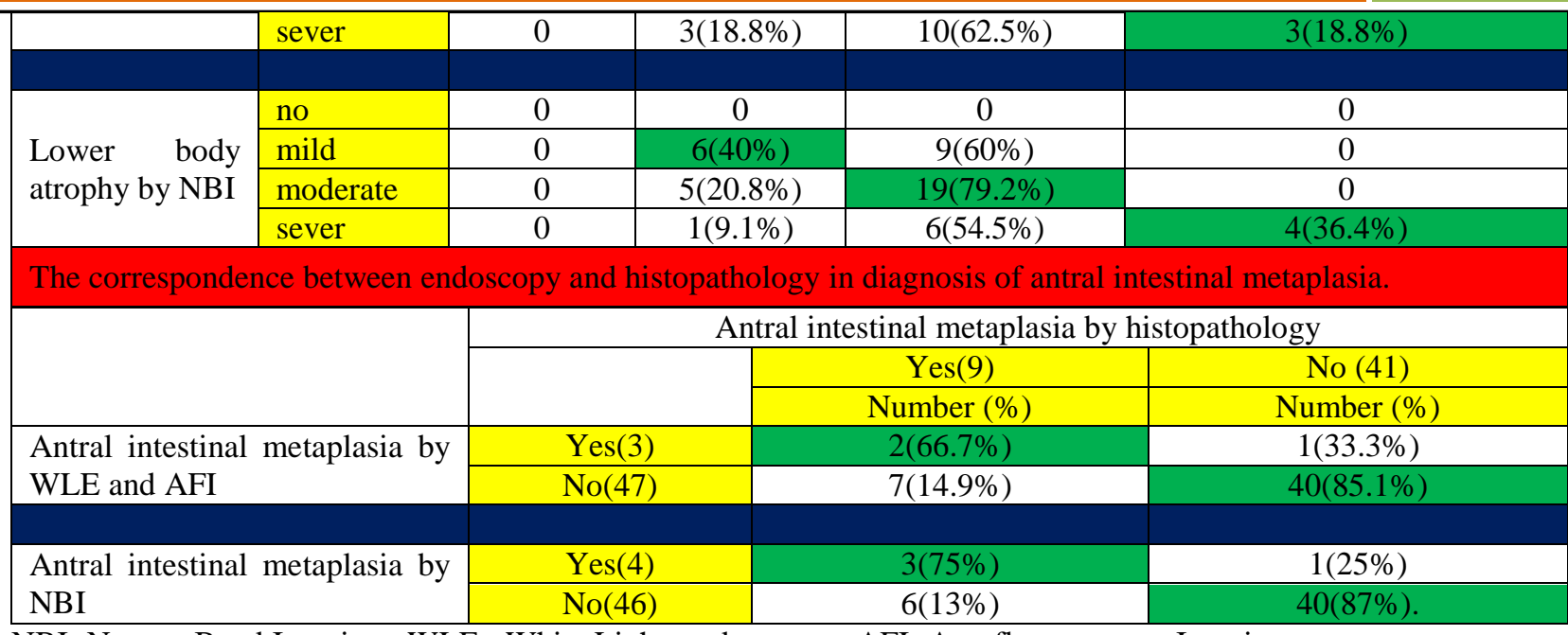

NBI=Narrow Band Imaging; WLE= White Light endoscopy ; AFI=Autofluorescence Imaging

Table 4 : The correspondence between NBI, WLE, and AFI stages and histopathology stages.

\begin{tabular}{|c|c|c|c|c|c|}
\hline \multirow{3}{*}{ Histopathology Stage } & \multicolumn{5}{|c|}{ Narrow band imaging stage } \\
\hline & Stage 0 & Stage I & Stage II & Stage III & Stage IV \\
\hline & $\begin{array}{c}\text { Number } \\
\%\end{array}$ & $\begin{array}{c}\text { Number } \\
\%\end{array}$ & $\begin{array}{c}\text { Number } \\
\%\end{array}$ & $\begin{array}{c}\text { Number } \\
\%\end{array}$ & $\begin{array}{c}\text { Number } \\
\%\end{array}$ \\
\hline Stage 0 & 0 & 0 & 0 & 0 & 0 \\
\hline Stage I & 0 & $6(54.5 \%)$ & $4(36.4 \%)$ & $1(9.1 \%)$ & 0 \\
\hline Stage II & 0 & $7(23.3 \%)$ & $16(53.3 \%)$ & $7(23.3 \%)$ & 0 \\
\hline Stage III & 0 & 0 & $2(33.3 \%)$ & $4(66.7 \%)$ & 0 \\
\hline Stage IV & 0 & 0 & 0 & 0 & $3(100.0 \%)$ \\
\hline \multicolumn{6}{|c|}{ Correspondence between WLE, and AFI stages and histopathology stages } \\
\hline \multirow{3}{*}{ Histopathology Stage } & \multicolumn{5}{|c|}{ WLE and AFI } \\
\hline & Stage 0 & Stage I & Stage II & Stage III & Stage IV \\
\hline & Number $\%$ & $\begin{array}{c}\text { Number } \\
\%\end{array}$ & $\begin{array}{c}\text { Number } \\
\%\end{array}$ & $\begin{array}{c}\text { Number } \\
\%\end{array}$ & $\begin{array}{c}\text { Number } \\
\%\end{array}$ \\
\hline Stage 0 & 0 & 0 & 0 & 0 & 0 \\
\hline Stage I & $4(36.4 \%)$ & $4(36.4 \%)$ & $1(9.1 \%)$ & $2(18.2 \%)$ & 0 \\
\hline Stage II & $3(10.0 \%)$ & $10(33.3 \%)$ & $7(23.3 \%)$ & $9(30 \%)$ & $1(3.3 \%)$ \\
\hline Stage III & 0 & $2(33.3 \%)$ & $2(33.3 \%)$ & $1(16.7 \%)$ & $1(16.7 \%)$ \\
\hline Stage IV & 0 & 0 & 0 & 0 & $3(100.0 \%)$ \\
\hline
\end{tabular}

WLE : white light endoscopy AFI : Autofluorescence Imaging

Table 5 : The concordance of gastric cancer risk assessment between histopathology and endoscopy.

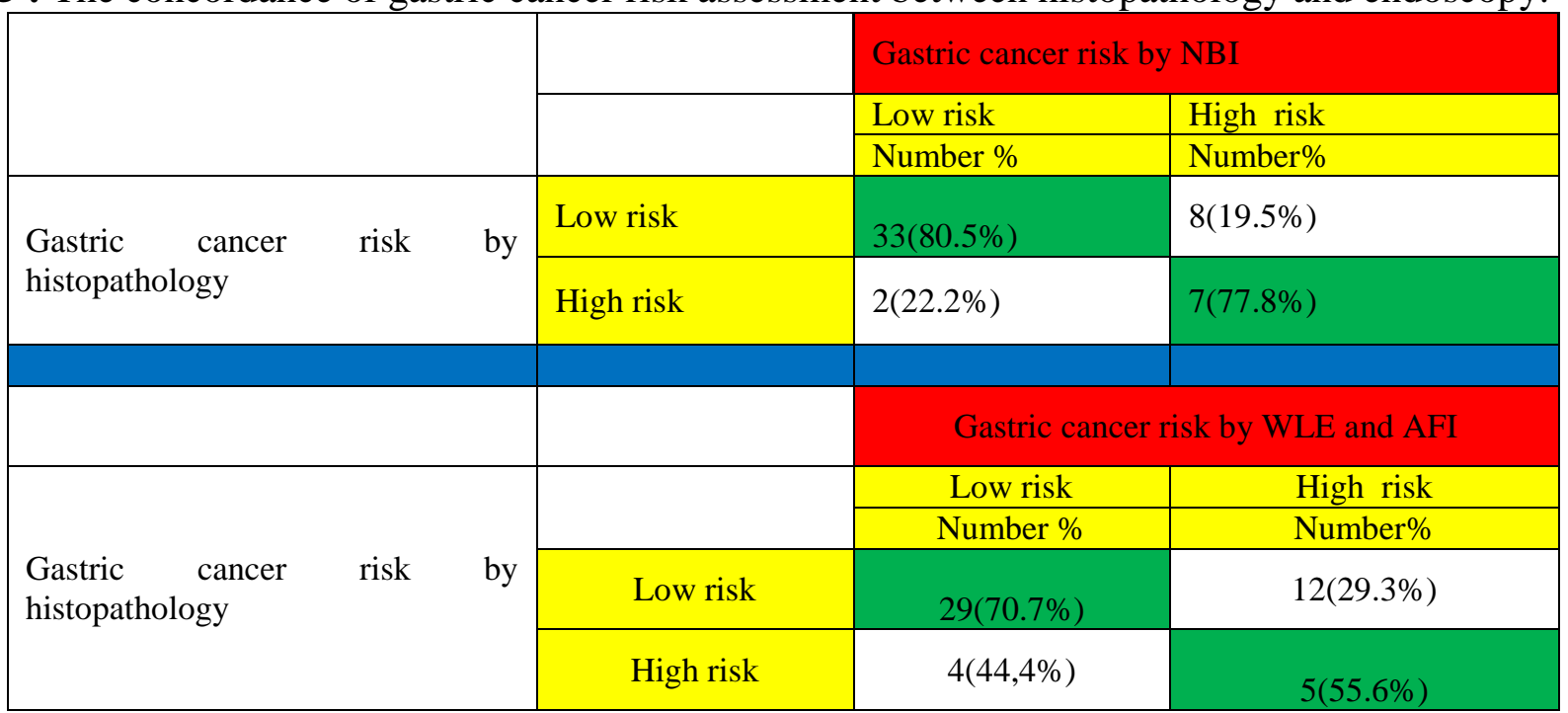

NBI=Narrow Band Imaging; WLI= White Light Imaging; AFI=Autofluorescence Imaging 
Figure 1 . Summary of the endoscopic findings of gastric atrophy scores

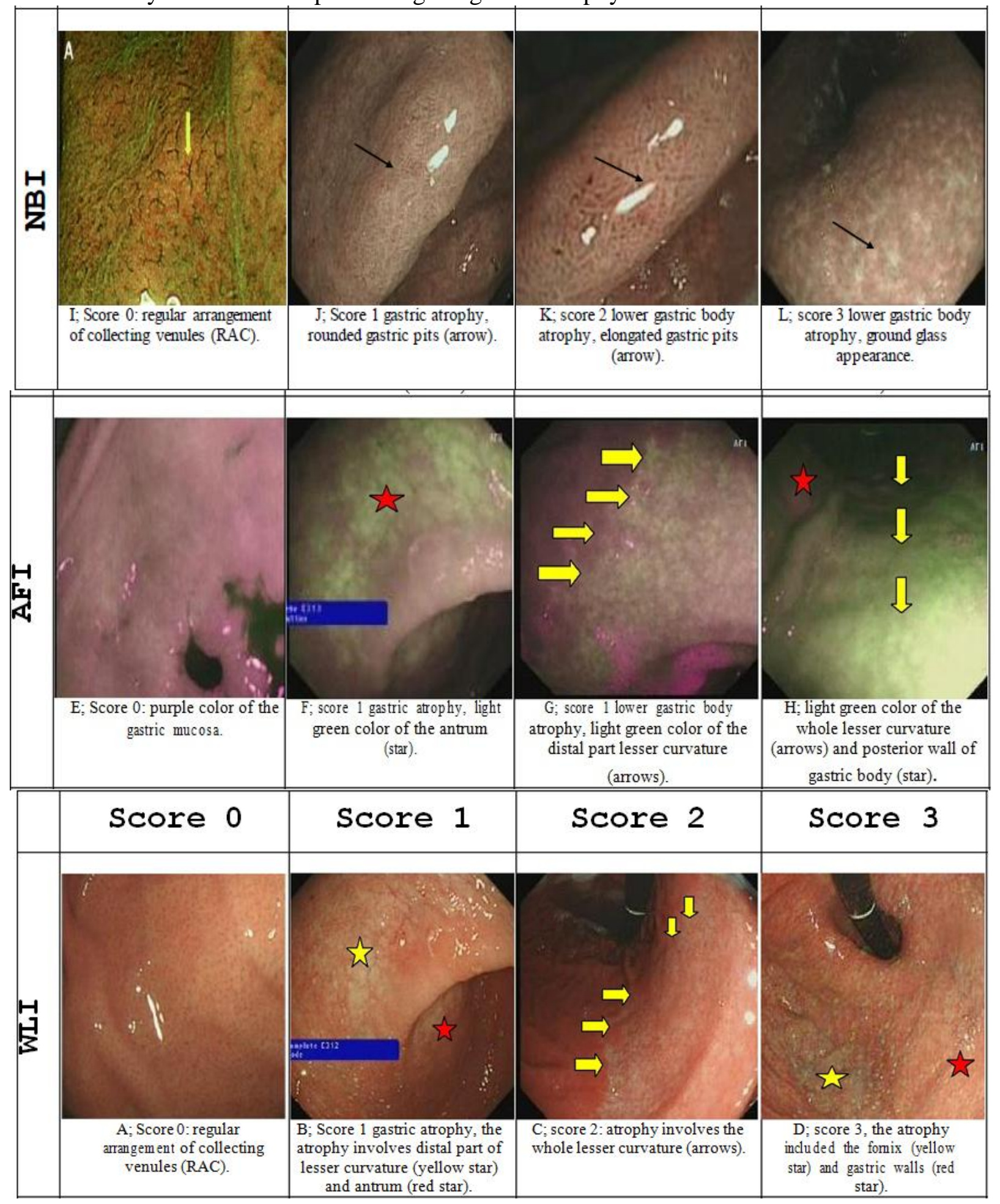

WLI (wight light image), NBI (narrow band image), AFI (Autofluorescence image ). 
Figure 2 . OLGA Score for lower gastric body atrophy .

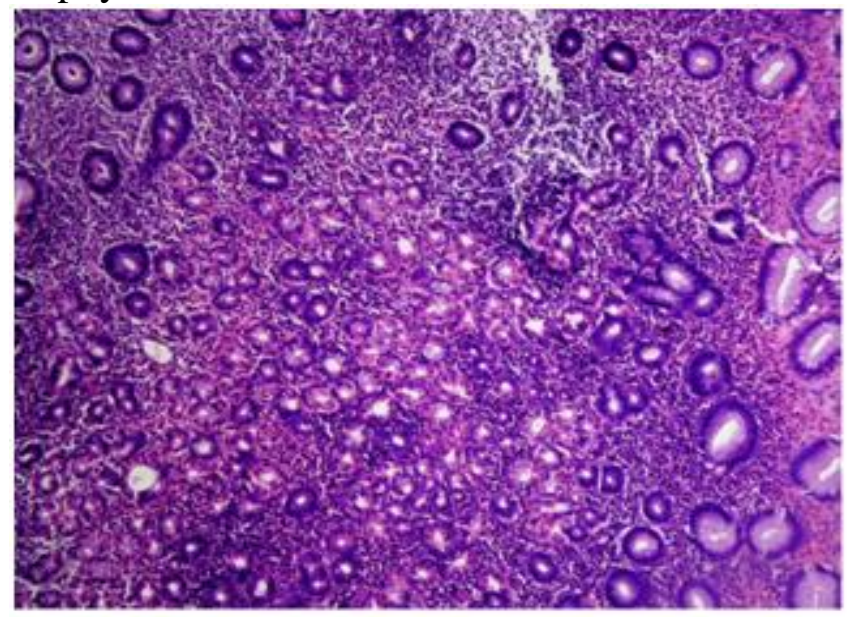

OLGA Score 1

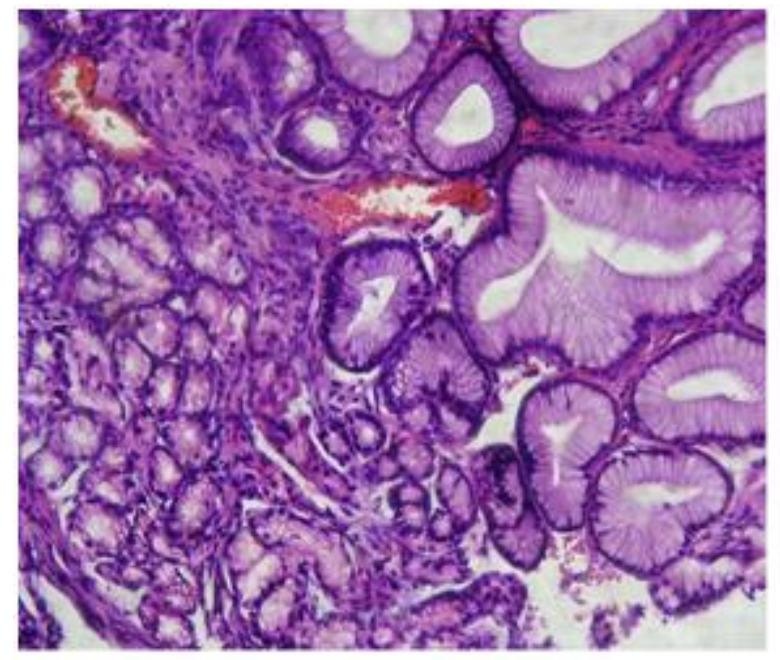

(B) OLGA Score 2

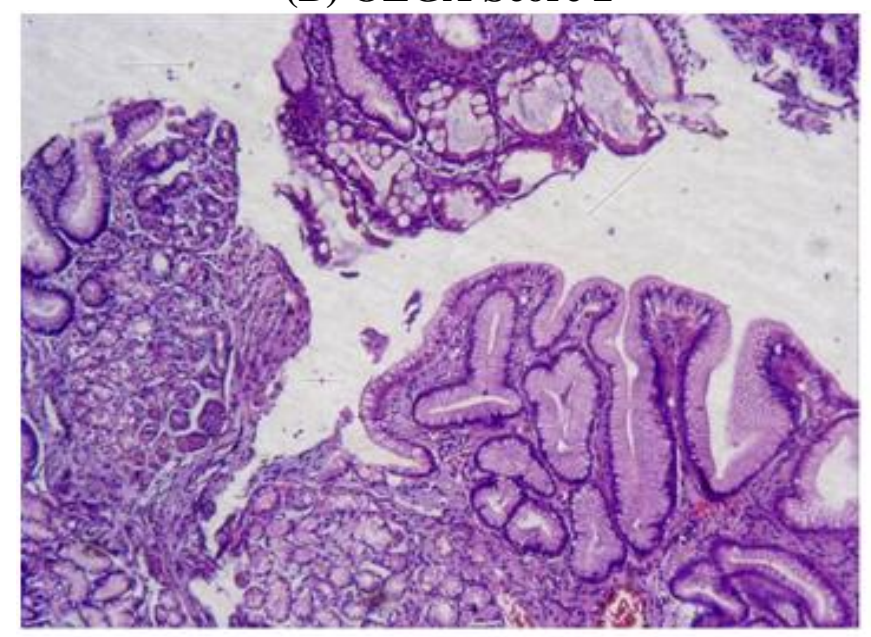

(c) OLIGA score 3

a) Body gastric mucosa showing only atrophy with no pseudopyloric gland metaplasia or intestinal metaplasia (score 1) OLGA and OLGIM staging system for atrophy (H\&E X 100). b) Body gastric mucosa showing prominent pseudopyloric gland metaplasia of the body glands (score 2) OLGA and OLGIM staging system for atrophy (H\&E X 200).

c) Body gastric mucosa showing pseudopyloric gland metaplasia and intestinal metaplasia (score 3) OLGA and OLGIM staging systems for atrophy (H\&E X200).

\section{Discussion}

Gastric cancer is associated with high morbidity and mortality. Atrophic gastritis and IM are well known risk factors for gastric cancer. Compared with other risk factors atrophic gastritis and IM markedly increases the gastric cancer risk ${ }^{[13]}$. A nationwide cohort study in the Netherlands showed that; the risk of gastric cancer increased in a step-wise manner according to the severity of gastric premalignant lesions.Within 5 years after diagnosis, the annual incidence of gastric cancer was $0.1 \%$, $0.25 \%, 0.6 \%$, and $6 \%$ for atrophic gastritis, IM, mild to moderate dysplasia and severe dysplasia, respectively ${ }^{[4]}$. Key points in the prevention and early detection of gastric cancer are to timely diagnose atrophic gastritis and IM, and to stratify gastric cancer risk according to their severity. The golden standard for diagnosing atrophic gastritis and intestinal metaplasia is the histological study of gastric mucosa. In fact Sydney gastritis classification provides little prognostic information and risk categorization for patients with gastritis. In 2007 an international group of pathologists proposed new gastritis histology to predict gastric cancer risk ${ }^{[14]}$. Later on the European guidelines suggested that systems for histopathological staging like operative link for gastritis assessment (OLGA) and operative link for gastric intestinal metaplasia (OLGIM) assessment may be useful for risk categorization of gastritis progression to gastric cancer ${ }^{[15]}$. In countries with high risk like South Korea, high OLGA and OLGIM stages were identified as independent risk factors for gastric cancer ${ }^{[16]}$. Rugge et al., $2010^{[17]}$, reported that in OLGA and OLGIM staging 0, I, and II, no neoplastic lesions were detected, and they were all clustered in stages III and IV. However, the invasive 
nature of gastric biopsy precludes its use for population screening. Moreover gastric biopsy cannot be performed in every patient. Many elderly patients have a risk of bleeding if stomach biopsy is attempted. On the other hand, withdrawal of antithrombotic agents to avoid biopsy-related bleeding may lead to thrombosis. Thus, taking several biopsy specimens for gastritis staging tends to be avoided. Furthermore, this approach for gastritis staging imposes a considerable burden on endoscopist, pathologist and substantially increases medical costs and procedure time ${ }^{[18]}$. Taken together these data suggested the importance of real time diagnosis of atrophic gastritis as well as IM and staging of gastric cancer risk, during endoscopic surveillance for gastric precancerous lesions. Recently Saka et al., concluded that staging of gastritis using NBI-magnifying endoscopy is able to approximate that determined by OLGA and OLGIM staging and is expected to be a practical approach that can replace staging based on the histology of biopsy specimen ${ }^{[18]}$. However examining the whole stomach by NBI-ME is difficult, time consuming, need special patient preparation and not suitable for patients screening. Thus precise and close examination by conventional endoscopy (WLE, AFI or NBI) without magnification may be more practical for screening purposes. In the current study we evaluated whether gastritis staging based on the OLGA staging systems using conventional (WLE, AFI, and NBI) endoscopy is equivalent to that determined by histology. We have classified gastric atrophy of the gastric corpus mucosa using Alaboudy et al., $2011^{[10]}$, classification with little modification to meet the OLGA scoring system. Also we depicted intestinal metaplasia in both gastric body and antrum as present or absent. We then obtained the scores based on conventional endoscopy (WLE, AFI, and NBI) and histopathology and tested the correspondence between them. The scores correspondence rates between combined WLE, AFI and NBI on one hand and OLGA score on the other hand were $28 \%$ and $58 \%$ for the corpus and $84 \%, 86 \%$ for the antrum, respectively. The rate of correspondence between the scores based on NBI and those based on histopathology were higher for scores 0 , I and III. On the other hand the rate of correspondence between the scores based on WLE and AFI, and those based on histopathology were higher for score II. This hint that gastric body mucosa with no or mild atrophy and that with severe atrophy with IM are diagnosed better with NBI, while that with moderate atrophy without intestinal metaplasia is diagnosed better with WLE and AFI. Although WLE, AFI, and NBI can exclude intestinal metaplasia with good rate in the gastric body it equally detected IM in 50\% of patients with gastric body IM. The gastritis stages were then decided according to the scores obtained by endoscopy and histopathology and divided into low risk (stages 0, I and II) and high risk (stages III and IV) groups. The rate of correspondence between WLE, AFI, and NBI and histopathology were $68 \%, 68 \%$, and $80 \%$ respectively. Although all modalities can detect patients with high risk accurately, the NBI rate of detection for patients with low risk was significantly higher than WLE and AFI. Many diagnostic endoscopic methods are used for improving detection of gastric premalignant lesions. Chromoendoscopy and magnifying endoscopy are the most popular worldwide. In the current study we suggested new endoscopic method for gastritis staging, using WLI, AFI and NBI to approximate the results of histopathology. WLE and AFI assessment of gastrointestinal tract reveals that this technique can be used to differentiate normal and abnormal mucosa. However the specificity of AFI is low. In the current study we found that the outcome of AFI examination is quite similar to that of WLE in assessment of lower gastric body atrophy and intestinal metaplasia of lower body and antrum. In contrast NBI enables detailed examination of the mucosal surface structure and vascular patterns, and hence precise characterization of the mucosal abnormality. According to our results NBI provided good diagnostic ability in categorization of risky patients into low and high risk groups, compared with WLE and AFI. Although NBI had good specificity in identification of patients with low risk and high risk for gastric cancer $(83 \%$ and $100 \%$, respectively), it had low specificity in prediction of 
patients with high risk for gastric cancer $(27 \%, \mathrm{n}=$ $3 / 11$ ). We think that this low specificity may be related to the small sample size and the limited familiarity with our new staging system; we believe that the specificity will increase with the increased number of tested patients and improved familiarity with our new staging system.

Author Disclosures: author declared that they have no conflicts of interest or financial ties to disclose.

\section{References}

1. Ferlay J, Soerjomataram I, Ervik M, et al .GLOBOCAN 2012 v1.0, Cancer Incidence and Mortality Worldwide: IARC Cancer Base No. 11 [Internet].Lyon, France: International Agency for Research on Cancer; 2013. Available from: http://globocan.iarc.fr, accessed on day/month/year.

2. Soetikno R, Kaltenbach $\mathrm{T}$, Yeh $\mathrm{R}$, et al. Endoscopic mucosal resection for early cancers of the upper gastrointestinal tract. $\mathbf{J}$ Clin Oncol.2005;23:4490-8.

3. Correa P. Chronic gastritis as a cancer precursor. Scand J Gastroenterol Suppl. 1984;104:131-136.

4. de Vries AC, van Grieken NC, Looman CW, Casparie, et al . Gastric cancer risk in patients with premalignant gastric lesions: a nationwide cohort study in the Netherlands. Gastroenterology. 2008;134:945-952.

5. Areia M, Carvalho R, Cadime AT, Rocha Gonçalves F, Dinis-Ribeiro M.et al., (2013). Screening for gastric cancer and surveillance of premalignant lesions: a systematic review of cost-effectiveness studies. Helicobacter; 18:325-337.

6. Rugge M, Kim JG, Mahachai V, et al. OLGA gastritis staging in young adults and country-specific gastric cancer risk. Int $\mathrm{J}$ Surg Pathol. 2008; 16: 150-154.

7. Capelle LG, de Vries AC, Haringsma, et al. The staging of gastritis with the OLGA system by using intestinal metaplasia as an accurate alternative for atrophic gastritis.
Gastrointestinal

Endoscopy.

$2010 \quad$;

71:1150-1158.

8. Kawamura M, Abe S, Oikawa K, et al . Topographic differences in gastric micromucosal patterns observed by magnifying endoscopy with narrow band imaging. J Gastroenterol Hepatol. 2011;26:477-483.

9. Kimura $\mathrm{K}$ and Takemoto T. An endoscopic recognition of the atrophic border and its significance in chronic gastritis.Endoscopy1969; 3:987-979.

10. Alaboudy A, Elbahrawy A, Matsumoto S , Yoshizawa A . Conventional Narrow-Band Imaging Has Good Correlation with Histopathological Severity of Helicobacter pylori Gastritis . Dig Dis Sci. 2011 ; 56:1127-1130.

11. Yao K . Light Blue Crests (LBCs) and White Opaque Substance (WOS).Zoom Gastroscopy. 2014; Japan: Springer; 73-81.

12. So J, Rajnakova A, Chan YH, Tay A, Shah $\mathrm{N}$, Salto-Tellez M, et al. Endoscopic trimodal imaging improves detection of gastric intestinal metaplasia among a high-risk patient population in Singapore. Dig Dis Sci. 2013;58:3566-3575. doi:

13. Yoon H, Kim N . Diagnosis and management of high risk group for gastric cancer. Gut Liver. 2015 ; 9(1):5-17. doi: 10.5009/gnl14118.

14. Rugge M, Meggio A, Pennelli G, et al. Gastritis staging in clinical practice: the OLGA staging system. Gut. 2007;56:631636. doi: 10.1136/gut.2006.106666.

15. Dinis-Ribeiro M, Areia M, de Vries AC, et al. Management of pre-cancerous conditions and lesions in the stomach (MAPS): guideline from the European Society of Gastrointestinal Endoscopy (ESGE), European Helicobacter Study Group (EHSG), European Society of Pathology (ESP), and the Sociedade Portuguesa de Endoscopia Digestiva (SPED) Endoscopy. 2012;44:74-94. doi: 10.1055/s-00311291491. 
16. Cho SJ, Choi IJ, Kook MC, et al. Staging of intestinal- and diffuse-type gastric cancers with the OLGA and OLGIM staging systems. Aliment Pharmacol Ther. 2013;38: 1292-1302. doi: 10.1111/apt.12515.

17. Rugge M, de Boni M, Pennelli G, et al. Gastritis OLGA-staging and gastric cancer risk: a twelve-year clinicopathological follow-up study. Aliment Pharmacol Ther. 2010;31:1104-1111.

18. Saka A, Yagi K and Nimura S. OLGA- and OLGIM-based staging of gastritis using narrowband imaging magnifying endoscopyDigestive Endoscopy 2015; 27: 735-742. 